\title{
ENGEVISTA
}

Página da revista: http://www.uff.br/engevista/seer/

\section{Estudo energético e econômico do aproveitamento do biogás \\ de um aterro sanitário em uma turbina a gás de 5,4 MW}

\section{Energy and economic study of the use of landfill biogas in a gas turbine of 5.4 MW}

\author{
Ielon Oliveira Pereira \\ Ivan Felipe Silva Santos \\ Rafaela Ediene Santos
}

Resumo: O aproveitamento energético de resíduos sólidos é uma das soluções tanto para disposição dos resíduos sólidos como para a geração de energia de modo alternativo e descentralizado. No Brasil, a Politica Nacional de Resíduos Sólidos incentiva o fim dos lixões, o que tem ocasionado um aumento do número de aterros sanitários no país, o que faz de surgir à oportunidade de aproveitamento do biogás destes aterros para geração elétrica. Neste contexto, uma análise energética e econômica do aproveitamento energético do biogás aterro sanitário da cidade de São José dos Campos (SP) por meio de uma turbina a gás foi conduzida. Os cálculos foram realizados por meio dos cálculos termodinâmicos da turbina e dados de custos da agência americana de proteção ambiental (USEPA). A energia produzida pelo empreendimento poderia suprir $11 \%$ do consumo residencial da cidade estudada e os resultados obtidos demonstram sua viabilidade econômica. Palavras-chave: Aterros sanitários; eletricidade; turbina a gás; viabilidade econômica.

\begin{abstract}
The energy use of solid wastes is one of the solutions both to solid waste disposal as to decentralized and alternative energy generation. In Brazil, the National Policy of Solid Wastes encourages the end of dumps, what has resulted in a grown in the number of sanitary landfills in the country. This growing establishes the opportunity of the use of the biogas from these landfills to electric generation. In this context, an energetic and economic analysis of the energy harnessing of the landfill gas of São José dos Campos (SP, Brazil) by means of a gas turbine were conducted. The calculations were done using a thermodynamic model applied to the studied turbine and data of costs from the United States Environmental Protection Agency (USEPA). The energy produced in the venture could supply $11 \%$ of city residential electric consumption and the obtained results shows the economic feasibility of the enterprise.
\end{abstract}

Keywords: Sanitary landfill; electricity; gas turbine; economic feasibility 


\section{Introdução}

O Brasil tem apresentado nos últimos um crescimento na geração de resíduos que pode ser verificada nas estimativas da Associação Brasileira de Empresas de Limpeza Pública e Resíduos Especiais (ABRELPE, 2015), que apresentou um crescimento de 215.297 para 218.874 [t/dia] (1,7\%) na geração de RSU entre 2014 e 2015, demonstrando um crescimento da geração de resíduos superior ao crescimento populacional no país no mesmo período $(0,8 \%)$.

Apesar de relacionado com o desenvolvimento econômico, o crescimento de geração de resíduos acarreta problemas que implicam em impactos socioeconômicos e ambientais, tais como: maior consumo de energia para tratamento e disposição, maior necessidade de área, gastos mais elevados, prejuízos para a saúde pública e meio ambiente quando os resíduos são dispostos de modo inadequado, etc. Além disto, como afirmam Johari et al. (2012), o gerenciamento dos resíduos domésticos está entre os principais desafios das economias em crescimento, ao lado de problemas globais, como a emissão de gases de efeito estufa. Estes fatores demonstram a necessidade de uma gestão eficiente de resíduos, que auxilie a combinação da solução da problemática da diminuição de geração e disposição adequada de resíduos e mantenha o desenvolvimento econômico.

No Brasil, a gestão de resíduos é estabelecida pela Politica Nacional de Resíduos Sólidos (PNRS, 2010). Uma das ações desta politica é o programa 'Brasil sem Lixões' que estabelecia o fim dos lixões como forma de disposição dos resíduos sólidos municipais do país até 2014. A meta não foi atingida no prazo dado, e a prorrogação deste limite está sendo discutida no Senado (BRASIL, 2017). Com a eliminação dos lixões, o numero de aterros sanitários tende a crescer, dado que esta é a alternativa mais barata em face às demais (Agamuthu, 2013). Até 2015, 58,7\% dos resíduos sólidos urbanos produzidos no Brasil eram enviados para aterros sanitários (ABRELPE, 2015).

Com o crescimento do número de aterros aumenta a oportunidade de recuperação de biogás nestes para geração elétrica. Conforme demonstra Leme et al. (2014), a implantação de usinas para geração de energia elétrica no aterro sanitário, diminui significativamente os impactos ambientais negativos do mesmo. As metas do plano nacional de resíduos sólidos (BRASIL, 2012), estabelecem uma potência instalada a partir do biogás de $250 \mathrm{MW}$ até 2031. De acordo com Santos et al. (2018a), o potencial total pode alcançar até 1800 MW.

As principais tecnologias de geração elétrica a partir do biogás são: i) Motores a combustão interna (MCI); ii) Turbinas a gás (TG) e iii) Microturbinas a gás (MTG - para potências de até $100 \mathrm{~kW}$ - Pecora, 2006). Apesar de mais caras que os motores de combustão interna (MCI), as TG são melhores ambientalmente e apresentam emissões de $\mathrm{NO}_{\mathrm{x}}$ bem inferiores as do MCI (Em casos extremos, chegando até a 100x inferiores - Pecora, 2006). Neste contexto, é avaliado energética e economicamente o aproveitamento do biogás do aterro 
sanitário da cidade de São José dos Campos (SP) para geração elétrica em uma turbina a gás, aplicando-se um modelo termodinâmico a uma turbina a gás comercial.

\section{Metodologia}

A cidade estudada foi de São José dos Campos, localizada na região sudeste do Brasil (Região mais prospera do país), no estado de São Paulo, a qual ocupa uma área de $1100 \mathrm{~km}^{2}$ (PMSJC, 2017). Os dados de população e geração de resíduos da cidade foram utilizados para predição da vazão de biogás e estudo energético e econômico da conversão elétrica do biogás produzido no aterro sanitário da cidade de São José em uma turbina a gás comercial. Os tópicos abaixo apresentam detalhadamente os passos necessários para os cálculos realizados.

\subsection{Projeção de resíduos e predição da produção de gás no aterro sanitário}

Adotou-se o modelo de taxa de crescimento decrescente para análise da projeção populacional. Tal modelo assume que a população de interesse possui um valor limite ou um ponto de saturação, não apresentando, portanto crescimento indefinido e é aplicado por meio das seguintes equações (1 a 3) (Qasim, 1999):

$$
\begin{gathered}
P_{d}=\frac{\ln \left[\left(P_{s}-P_{2}\right) /\left(P_{s}-P_{1}\right)\right]}{t_{1}-t_{2}} \\
P_{S}=\frac{2 \cdot P_{0} \cdot P_{1} \cdot P_{2}-P_{1}{ }^{2}\left(P_{0}+P_{2}\right)}{P_{1} \cdot P_{2}-P_{1}{ }^{2}} \\
P(t)=P_{2}+\left(P_{s}-P_{2}\right) \cdot\left(1-e^{-P d\left(t-t^{2}\right)}\right)
\end{gathered}
$$

Onde: $\mathrm{P}_{\mathrm{s}}=$ Ponto de saturação; $\mathrm{P}_{\mathrm{d}}=$ Constante de proporcionalidade; índices 0,1 e 2: relativos aos três anos de dados históricos utilizados na análise (Dados estes obtidos em IBGE, 2017), $t=$ ano desejado e $P_{t}=$ População estimada no ano $t$.

Para obter-se a quantidade de resíduos produzidos utilizou-se o índice de geração de resíduos de 0,86 kg/habitante.dia, retirado do Sistema Nacional de Informações sobre Saneamento (SNIS, 2016) para o ano de 2015, considerando um aumento de $1 \%$ ao ano. (Barros, 2012). A partir da projeção de coleta de resíduos sólidos, é possível calcular a geração total de resíduos anual, em toneladas, através da equação 4.

$$
R_{t}=\frac{P_{t} \cdot I_{g} \cdot 365}{1000}
$$

Onde: $R_{t}=$ geração de resíduos no ano $t$ em toneladas; $P_{t}=$ população no ano $t$ e $I_{g}=$ índice de coleta de resíduos no ano t em $\mathrm{kg} / \mathrm{habitante.dia.}$

A produção de biogás foi estimada pelo software LandGEM®. Este é um modelo de cinética de primeira ordem desenvolvido pelo centro de controle tecnológico (Control Technology Center - CTC) da agência de proteção ambiental americana (USEPA) em 2005 (Equação 5- USEPA, 2005).

$$
Q_{L}=\sum_{i=1}^{n} \sum_{j=0,1}^{1} k \cdot L_{0} \cdot\left(\frac{W_{L i}}{10}\right) \cdot e^{-k t_{i j}}
$$


Onde: $\mathrm{k}=$ taxa de decaimento da produção de metano em $1 /$ ano, $\mathrm{L}_{0}=$ potencial de geração de metano em $\mathrm{m}^{3} / \mathrm{t}, \mathrm{W}_{\mathrm{Li}}=$ massa de resíduo enviada ao aterro no ano i e $\mathrm{Q}_{\mathrm{L}}=$ vazão anual de biogás $\mathrm{m}^{3} /$ ano.

Os dados da geração de resíduos calculados pela equação 4 foram inseridos no software LandGem ${ }^{\circledR}$, para previsão da produção de gás no aterro. Os parâmetros $\mathrm{k}$ e $\mathrm{L}_{0}$ foram selecionados utilizando-se dados do Banco Mundial (2004) considerando-se a precipitação anual média da cidade escolhida (1155 mm - Instituto Nacional de Meteorologia, 2015). Sendo adotados como: 0,06 L/ano e $170 \mathrm{~m}^{3} / \mathrm{t}$. O software aplicou então a equação 5 para previsão da produção de gás no aterro analisado. Foi adotado um valor de eficiência de coleta do gás do aterro de 75\% (Barros, 2012).

\subsection{Modelo termodinâmico e cálculos energéticos}

Para a simulação da geração de energia a partir do biogás, foram utilizados os dados da turbina cujo modelo SGT-100 (SIEMENS, 2012) tem capacidade máxima de 5,4 MW, sendo:

- Coeficiente de pressão do compressor e da turbina: 15,6:1.

- Fluxo de gás de escape: $20,6 \mathrm{~kg} / \mathrm{s}$.

- Temperatura adiabática de chama: $1000{ }^{\circ} \mathrm{C}$

- Temperatura: $531^{\circ} \mathrm{C}$.

A Tabela 1 as faixas de variação do percentual de metano do biogás. Este é responsável pelo poder calorifico e potencial energético do gás a ser queimado. Adotou-se um percentual de $62,5 \%$.

A base de cálculos da potência útil para a turbina SGT-100 foi construída em Excel com ativação do suplemento COOLPROP. Este banco de dados possui diversas propriedades dos fluidos, dentre elas as propriedades termodinâmicas, tal como: entalpia, entropia, pressão, entre outras.

Tabela 1: Composição do Biogás. Fonte: European Biomass Association (AEROBIOM, 2009).

\begin{tabular}{|c|c|}
\hline Componentes & Percentual em volume (\%) \\
\hline Metano $\mathrm{CH}_{4}$ & $50-75$ \\
\hline Dióxido de Carbono $\mathrm{CO}_{2}$ & $24-45$ \\
\hline Vapor d'água $\mathrm{H}_{2} \mathrm{O}$ & $1-2$ \\
\hline Monóxido de Carbono CO & $0-0,3$ \\
\hline Nitrogênio $\mathrm{N}_{2}$ & $1-5$ \\
\hline Gás Sulfídrico $\mathrm{H}_{2} \mathrm{~S}$ & $0,1-0,5$ \\
\hline Hidrogênio $\mathrm{H}_{2}$ & $0-3$ \\
\hline Oxigênio $\mathrm{O}_{2}$ & - \\
\hline
\end{tabular}

O limite para calcular a temperatura de chama dentro da margem de acerto do COOLPROP para os gases de exaustão, sendo eles: nitrogênio, oxigênio, dióxido de carbono e vapor de água, é de $2000 \mathrm{~K}$. Utilizando este recurso, é possível alterar os parâmetros iniciais do modelo matemático, como: a massa de combustível e os dados da turbina. Com isto, é possível 
otimizar e ajustar os resultados, e até mesmo simular outras potências com outras composições, deste combustível orgânico, aliados à outros modelos de turbinas a gás. As equações aqui aplicadas para cálculo do modelo termodinâmico foram obtidas em Moran et al. (2013).

O conceito fundamental para gerar o modelo, citado acima, parte da análise do processo de combustão. A primeira coisa a se verificar em uma reação é o balanceamento de massa e energia através da equação estequiométrica que podem ser expressas da seguinte forma: Reagentes $\rightarrow$ Produtos, ou, Combustível + Oxidante $\rightarrow$ Produtos.

Considere que $1 \mathrm{kmol}$ de $\mathrm{H}_{2}$ seja combustível e $\mathrm{O}_{2}$ seja o oxidante, admitindo que a reação seja completa o resultado do balanceamento será conforme equação 6 .

$$
1 \mathrm{kmol} \mathrm{H}_{2}+0.5 \mathrm{kmol} \mathrm{O}_{2} \rightarrow 1 \mathrm{kmol} \mathrm{H}_{2} \mathrm{O}
$$

Para o caso dos motores, as reações acontecem com os hidrocarbonetos, que são cadeias entre os elementos químicos Hidrogênio e Carbono, como por exemplo, o próprio gás natural. Considere uma reação completa entre o $\mathrm{CH}_{4}$ (metano) a uma quantidade de ar, os produtos serão $\mathrm{CO}_{2}, \mathrm{H}_{2} \mathrm{O}$ e $\mathrm{N}_{2}$ (Equação 7):

$$
\mathrm{CH}_{4}+a\left(\mathrm{O}_{2}+3,76 \mathrm{~N}_{2}\right) \rightarrow b C \mathrm{O}_{2}+\mathrm{H}_{2} \mathrm{O}+d \mathrm{~N}_{2}
$$

Para se calcular a massa e posteriormente a quantidade de energia que será liberada, o sistema pode ser resolvido da seguinte forma:

$$
\begin{array}{cc}
C: & b=1 \\
H: & 2 c=4 \\
O: & 2 b+c=2^{a} \\
N: & d=3,76^{a}
\end{array}
$$

Resolvendo as equações o balanceamento será (Equação 8):

$$
\mathrm{CH}_{4}+2\left(\mathrm{O}_{2}+3,76 \mathrm{~N}_{2}\right) \rightarrow \mathrm{CO}_{2}+2 \mathrm{H}_{2} \mathrm{O}+7,52 \mathrm{~N}_{2}
$$

Dentro do processo de oxidação dos combustíveis também há uma possível e importante relação, que é feita dentro do balanço estequiométrico e que vai implicar diretamente na taxa de liberação de energia. É a razão ar/combustível (AC - equação 9) também conhecida como excesso de ar, ela é expressa através do quociente entre a massa de ar pela massa de combustível.

$$
A C=\frac{m_{a r}}{m_{\text {combustivel }}}
$$

Tomando os valores da Equação 8 como exemplo para calcular a sua relação AC. Sabendo que $\overline{\mathrm{AC}}$ é a razão combustível na base molar, e $\mathrm{M}_{\mathrm{i}}$ é Massa Molecular, então obtêm-se a equação 10. 


$$
\mathrm{AC}=\overline{\mathrm{AC}}\left(\frac{\mathrm{M}_{\mathrm{ar}}}{\mathrm{M}_{\text {combustivel }}}\right)=9,52\left(\frac{28,97}{16,04}\right)=17,19
$$

Em boa parte dos casos se a relação $\mathrm{AC}$ se for excessiva poderá haver quantidade a mais de oxigênio nos produtos, com o manuseio deste recurso a mistura pode-se chegar a misturas pobres ou ricas, interferindo diretamente na temperatura de chama. Na transferência de calor dos gases de combustão para o meio ambiente a maior temperatura alcançada no limite de um sistema adiabático do reator é chamada de temperatura adiabática de chama. Na prática isto não acontece devido às irreversibilidades de segunda Lei da Termodinâmica, a temperatura adiabática é apenas uma estimativa teórica. Para se calcular a temperatura de chama, é preciso estimar o balanço de energia entre os elementos químicos da reação conforme a Equação 11.

$$
\begin{gathered}
\sum_{P} n_{s}\left(\bar{h}_{f}^{o}+\Delta \bar{h}\right)_{s}=\sum_{R} n_{e}\left(\bar{h}_{f}^{o}+\Delta \bar{h}\right)_{e} \\
\overline{\mathrm{h}}_{\mathrm{i}}=\overline{\mathrm{h}}_{\mathrm{ref}}-\overline{\mathrm{h}}_{298}^{\mathrm{o}}
\end{gathered}
$$

Onde:

$\mathrm{n}_{\mathrm{s}}$ : número de moles do combustível na saída.

$\mathrm{n}_{\mathrm{e}}$ : número de moles do combustível na entrada.

$\overline{\mathrm{h}}_{\mathrm{f}}^{\mathrm{o}}$ : entalpia (específica) de formação.

$\Delta \overline{\mathrm{h}}$ : Variação de entalpia de produtos e reagentes.

Com o auxílio do CoolProp se encontra a entalpia de referência $\overline{\mathrm{h}}_{\text {ref }}$ dentro da planilha em Excel, dessa forma é possível conhecer ou interpolar a temperatura de chama, pois as propriedades fazem parte do banco de dados deste programa (COOLPROP, 2016).

Inicialmente, em um processo termodinâmico é necessário estabelecer um volume de controle, assim como, conhecer as grandezas que ali estarão envolvidas, ou seja, quanto de calor (Q) será utilizado para produzir um determinado trabalho (W). A energia será transferida pela massa que percorre o sistema, essa transferência ocorre devido à alteração dos valores das propriedades termodinâmicas onde a energia interna, potencial, cinética, térmica e o trabalho podem ser expressos pela Equação 13 (Pereira, 2018).

$$
\sum\left(\dot{\mathrm{Q}}_{\mathrm{vc}}-\dot{\mathrm{W}}_{\mathrm{vc}}\right)+\sum_{\mathrm{i}=\mathrm{e}=1}^{\mathrm{n}}\left(\dot{\mathrm{m}}_{\mathrm{e}} \mathrm{h}_{\mathrm{e}}-\dot{\mathrm{m}}_{\mathrm{s}} \mathrm{h}_{\mathrm{s}}\right)+\sum_{\mathrm{i}=\mathrm{e}=1}^{\mathrm{n}}\left(\mathrm{gz}_{\mathrm{e}-\mathrm{gz}}\right)+\sum_{\mathrm{i}=\mathrm{e}=1}^{\mathrm{n}}\left(\frac{\mathrm{V}_{\mathrm{e}}^{2}-\mathrm{V}_{\mathrm{s}}^{2}}{2}\right)=0
$$

Onde:

g - Aceleração da gravidade $\left[\mathrm{m} / \mathrm{s}^{2}\right]$;

$\mathrm{h}_{\mathrm{e}}$ - Entalpia específica na entrada do volume de controle $[\mathrm{kJ} / \mathrm{kg}]$;

$\mathrm{h}_{\mathrm{s}}$ - Entalpia específica na saída do volume de controle $[\mathrm{kJ} / \mathrm{kg}]$;

$\dot{\mathrm{m}}_{\mathrm{e}}$ - Fluxo de massa na entrada do volume de controle $[\mathrm{kg} / \mathrm{s}]$;

$\dot{\mathrm{m}}_{\mathrm{s}}$ - Fluxo de massa na saída do volume de controle $[\mathrm{kg} / \mathrm{s}]$; 
$\dot{\mathrm{Q}}_{\mathrm{vc}}$ - Taxa de calor para o volume de controle $[\mathrm{kW}]$;

Ve- Velocidade na entrada $[\mathrm{m} / \mathrm{s}]$;

Vs- Velocidade na saída $[\mathrm{m} / \mathrm{s}]$;

$\dot{\mathrm{W}}_{\mathrm{vc}}$ - Trabalho ou potência referente ao volume de controle $[\mathrm{kW}]$;

$\mathrm{z}_{\mathrm{e}}$ - Altura potencial na entrada $[\mathrm{m}]$;

$\mathrm{z}_{\mathrm{s}}$ - Altura potencial na saída [m].

De modo resumido, o balanço de massa e energia dentro de um volume de controle é dado por: Alterações no conteúdo $=+$ entrada - saída. Considerando-se que as energias: cinética e potencial são irrelevantes, a Equação 13 pode ser reescrita conforme a Equação 14 (Pereira, 2018).

$$
\sum\left(\dot{\mathrm{Q}}_{\mathrm{vc}}-\dot{\mathrm{W}}_{\mathrm{vc}}\right)+\sum_{\mathrm{i}=\mathrm{e}=1}^{\mathrm{n}}\left(\dot{\mathrm{m}}_{\mathrm{e}} \mathrm{h}_{\mathrm{e}}-\dot{\mathrm{m}}_{\mathrm{s}} \mathrm{h}_{\mathrm{s}}\right)=0
$$

Por esta equação obtêm-se as devidas grandezas energéticas da turbina a gás. O cálculo da eficiência elétrica de primeira lei para um ciclo termodinâmico $\left(\eta_{\mathrm{EL}}\right)$ pode ser expresso pela Equação 15.

$$
\eta_{\mathrm{EL}}=\frac{\dot{\mathrm{W}}_{\mathrm{EL}}}{\dot{\mathrm{Q}}_{\text {entrada }}}
$$

O termo trabalho do $\dot{\mathrm{W}}_{\mathrm{EL}} \mathrm{e}$ equivalente à potência elétrica $\left(\mathrm{P}_{\mathrm{el}}\right)$ do ciclo termodinâmico. Este termo pode ser utilizado para o cálculo da produção energética da turbina a gás conforme equação 16 (Santos et al., 2018b)

$$
E=P_{e l} \cdot f_{c} \cdot 8760
$$

Onde: $\mathrm{E}=$ energia elétrica e $\mathrm{f}_{\mathrm{c}}=$ fator de capacidade.

\subsection{Cálculos econômicos}

$\mathrm{Na}$ análise de viabilidade econômica foram considerados os parâmetros valor presente líquido (VPL) e o custo de eletricidade nivelada (LCOE), conforme as equações 17 e 18 (Branker et al., 2011), respectivamente. Se o VPL for positivo, verifica-se a viabilidade econômica do empreendimento. Caso contrário o empreendimento não é atraente economicamente. O LCOE, por sua vez, refere-se à razão entre a somatória dos custos transferidos ao ano inicial e a energia também descontada no tempo, e representa a tarifa mínima de venda de energia para que o empreendimento possua viabilidade econômica. Desta forma, se a tarifa de venda de energia for superior ao LCOE do empreendimento, há ocorrência de viabilidade econômica (Branker et al., 2011).

$$
V P L=\sum_{t=1}^{m} \frac{\left(E_{n} \times T\right)-C_{o \& m}}{(1+i)^{n}}-I
$$




$$
L C O E=\frac{\sum_{t=1}^{m} \frac{C_{n}}{(1+i)^{n}}}{\sum_{t=1}^{m} \frac{E_{n}}{(1+i)^{n}}}
$$

Onde: $\mathrm{E}_{\mathrm{n}}=$ energia produzida anualmente; $\mathrm{T}$ = tarifa de venda de energia, em USD/MWh - adotada igual a 280 R \$/MWh, valor mínimo do leilão A-6 para térmicas a gás da Agência Nacional de Energia Elétrica (ANEEL, 2018); $C_{o \& m}=$ custo de operação e manutenção; $\mathrm{i}=$ taxa de juros adotada como sendo $10 \%$ a.a.; $\mathrm{I}=$ investimento inicial; $\mathrm{m}=$ vida útil do projeto em anos; $\mathrm{n}=$ ano de análise e $\mathrm{C}_{\mathrm{n}}=$ custo do empreendimento em cada ano (igual ao investimento inicial no ano 0 e ao custo de operação e manutenção nos demais anos).

O investimento inicial (I) e o custo de operação em manutenção $\left(\mathrm{C}_{0 \text { om }}\right)$ foram calculados com base nas equações 19 e 20, desenvolvidas pela agência americana de proteção ambiental (USEPA, 2016) para sistemas de aproveitamento energético do biogás que considerem: i) Compressão e tratamento do gás; ii) Turbina e gerador elétrico; iii) Construções civis; iv) Equipamentos de coleta de gás e v) equipamentos de interconexão elétrica.

$$
\begin{gathered}
I=2340 \cdot P-0.103 \cdot P^{2}+250.000 \\
C_{O \& m}=0,0144 . E
\end{gathered}
$$

Onde: $\mathrm{I}=$ investimento em Dólares, $\mathrm{P}=$ potência em $\mathrm{kW}, \mathrm{E}=$ energia em GWh e Co\&m = custo de operação e manutenção em milhões de dólares. Uma taxa de conversão de USD para R\$ de 3,25 (Cotação do dia 21/02/2018 - INVESTING, 2018) e uma taxa de importação dos equipamentos de $15 \%$ do valor inicial foram consideradas (Taxa próxima ao valor obtido para importação de turbinas em BRASIL, 2018).

\section{Resultados}

\subsection{Resultados da projeção populacional e predição de biogás}

Utilizando as equações a 1 a 4 pode-se calcular a projeção populacional e a projeção de resíduos a cada ano na cidade analisada. Este resultado é apresentado na Figura 1. A partir dos dados de geração total de resíduos anual, o software LandGem® foi aplicado para cálculo da vazão de biogás produzida anualmente no aterro sanitário. A projeção de gás é apresentada na Figura 2, juntamente com a vazão coletada. 
ISSN: $1415-7314$

ISSN online: $2317-6717$

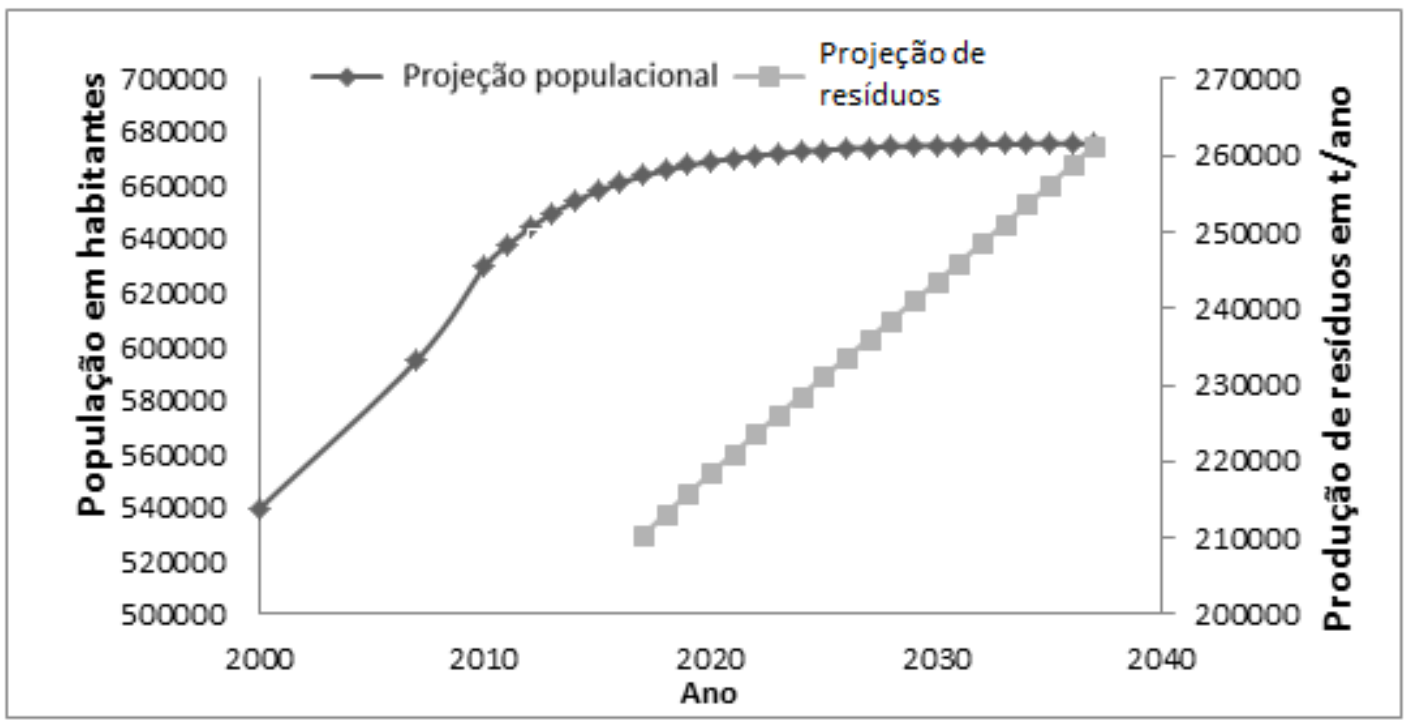

Figura 1: Projeção populacional e de produção de resíduos da Cidade de São José dos Campos.

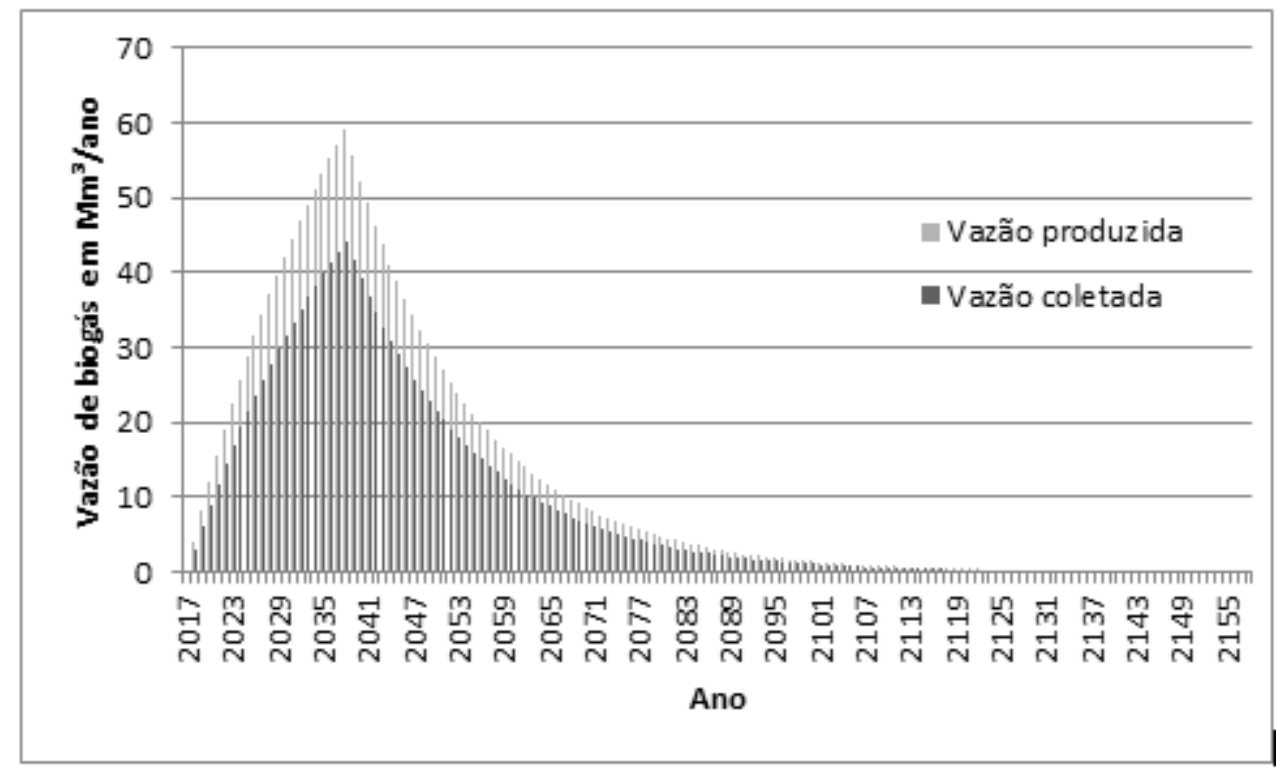

Figura 2: Produção de biogás no aterro sanitário.

A vazão de Biogás produzido no aterro a ser aproveitada foi definida a partir da turbina escolhida SGT100 (Siemens), sendo igual a $0,68 \mathrm{~m}^{3} / \mathrm{s}$ ou 21,4 Mm³/ano. Pela Figura 2 observase que esta vazão está disponível por 35 anos, entre os anos de 2025 e 2051. Será estudada a utilização deste biogás entre os anos de 2025 a 2040, totalizando um tempo de aproveitamento de 15 anos (Como adotado por Santos et al., 2018).

\subsection{Potência e eficiência}

Com Base nos dados obtidos do aterro sanitário da cidade de São José dos Campos (SP), foi possível configurar o modelo de turbina a gás para geração de energia com os seguintes dados de fábrica: 
- $\quad$ SGT100 (Siemens).

- $\quad$ Coeficiente de pressão do compressor 15,6:1.

- $\quad$ Fluxo de gás de escape $20,6 \mathrm{~kg} / \mathrm{s}$.

- Temperatura dos gases de exaustão $536{ }^{\circ} \mathrm{C}$.

Para modelar a potência desta turbina, com o biogás em questão, foi utilizado o software Microsoft Excel com ativação do suplemento COOLPROP, que aproxima as propriedades termodinâmicas, tais como entalpia e entropia. Ao informar, por exemplo: temperatura e pressão do combustível, automaticamente as demais propriedades que se deseja saber são calculadas e exibidas.

O código deve ser descrito, neste caso, dentro das células de Excel da seguinte maneira: =Props("H";"P";(pressão em bar);"T";(temperatura em Kelvin);"elemento químico"). Sendo: H: entalpia (neste caso a propriedade que se deseja encontrar para calcular o trabalho), P: pressão, T: temperatura e Elemento químico, pode ser: $\mathrm{CH}_{4}, \mathrm{CO}_{2}, \mathrm{~N}_{2}, \mathrm{O}_{2}$, entre outros. Os resultados obtidos do modelo termodinâmico aplicado estão apresentados na Tabela 2.

Tabela 2: Resultados do modelo gerado através do biogás

\begin{tabular}{|c|c|}
\hline Grandezas & Resultados \\
\hline Calor Produzido [MW] & 22,84 \\
\hline Energia Consumida [MW] & 7,28 \\
\hline Calor Rejeitado [MW] & 11,28 \\
\hline Potência Líquida [MW] & 4,28 \\
\hline Eficiência elétrica [\%] & 18,75 \\
\hline
\end{tabular}

Assim sendo, o calor produzido na câmara de combustão é de 22,84 MW, o trabalho do compressor é de 7,28 MW, o calor cedido para o meio ambiente é de 11,28 MW, potência útil 4,28 MW e a eficiência deste sistema é de 18,75\%. Tomando-se um fator de capacidade de $80 \%$ (como considerado por Santos et al., 2018a), pode-se calcular a energia passível de ser produzida pela Equação 16, que foi igual a $30 \mathrm{GWh} /$ ano. Sabendo-se que o consumo elétrico residencial médio no Brasil é de 2,04 MWh/ano (EPE, 2015), aproximadamente 15.000 residências poderiam ser supridas pela energia produzida no aterro. Se for assumida uma média de 4 habitantes por residência, um total de 60.000 habitantes poderão ser supridos. Este valor equivale a $11 \%$ do total de habitantes da cidade projetado para o ano de 2020.

\subsection{Resultados econômicos}

De posse dos cálculos energéticos e das equações apresentadas no tópico 2.3 pode-se calcular os parâmetros econômicos do empreendimento. Estes estão apresentados na tabela 3. Pelos resultados da tabela 3 observa-se a viabilidade do empreendimento de geração elétrica a partir do biogás em São José dos Campos. Comparando-se o resultado do LCOE (215 $\mathrm{R} \$ \mathrm{MWh}$ ) com a tarifa de venda de energia adotada (similar a de usinas térmicas a gás natural $280 \mathrm{R} \$ \mathrm{MWh}$ ), observa-se que a cada MWh comercializado pela usina, um lucro de $75 \mathrm{R} \$$ seria 
obtido. O custo unitário obtido de $8.00,00 \mathrm{R} \$ / \mathrm{kW}$ é ligeiramente superior aos custos de outras fontes renováveis como pequenas centrais hidrelétricas e eólicas (Cujo custo médio não supera 6.000,00 R $\$ / \mathrm{kW}$ - Rangel et al., 2016), mas ainda assim é inferior aos custos da energia solar fotovoltaica de larga escala (Em média superior a 10.000,00 R $\$ / \mathrm{kW}$ segundo Rangel et al., 2016). Vale ressaltar que o aproveitamento energético do gás de aterro é um aproveitamento baseado em resíduos, o que é incentivado pela Politica Brasileira de Resíduos Sólidos (PNRS, 2010) e de alto benefício ambiental, dado que estimula a implantação de aterros sanitários de maior qualidade ambiental e com maior controle de captura de gases (que deixam então de ser emitidos para atmosfera).

Tabela 3: Resultados dos cálculos econômicos

\begin{tabular}{|c|c|}
\hline Parâmetro & Valor \\
\hline Investimento em milhões de $\mathrm{R} \$$ & 38,3 \\
\hline Custo unitário em $\mathrm{R} \$ / \mathrm{kW}$ & $7.794,84$ \\
\hline Co\&m em Milhões de $\mathrm{R} / \mathrm{ano}$ & 1,40 \\
\hline VPL em Milhões de R\$ & 14,84 \\
\hline LCOE em R $\$ / \mathrm{MWh}$ & 215 \\
\hline
\end{tabular}

\section{Conclusões}

Foi avaliada, enérgica e economicamente, a utilização de turbinas a gás para geração de energia elétrica a partir do biogás de aterro sanitário. Para tal foi analisado o aterro sanitário da cidade de São José dos Campos (SP). O uso das turbinas a gás, apesar de mais caro que outras tecnologias, diminui as emissões de $\mathrm{NO}_{\mathrm{x}}$ contribuindo para uma melhor qualidade do ar na cidade.

A potência elétrica liquida resultante do empreendimento foi de 4,28 MW. A energia elétrica passível de ser produzida pelo empreendimento (30 GWh/ano) poderia suprir até $11 \%$ da população da cidade. Embora os custos do empreendimento sejam superiores a de outras renováveis como as pequenas centrais hidrelétricas e eólicas, a viabilidade econômica do empreendimento foi assegurada e um lucro de $75 \mathrm{R} \$$ seria obtido em cada MWh. Vale ainda ressaltar a importância destes empreendimentos para incentivar o setor de saneamento no Brasil, colaborando com a implantação de aterros de melhor qualidade de controle e menores impactos ambientais.

Foi utilizada uma taxa de desconto de $10 \%$ ao ano. Cada elevação de $1 \%$ desta taxa contribuiria para elevação de aproximadamente 9 R $\$ / M W h$ no LCOE, onerando o empreendimento. Outro fator que pode colaborar com a oneração do empreendimento é o aumento dos valores de taxa de importação de tecnologia, que teria impacto imediato sobre o custo inicial do projeto. Tais fatores apontam para necessidade de desenvolvimento de tecnologia nacional e de incentivos públicos para preservação e ampliação da viabilidade de empreendimentos de aproveitamento energético em aterros sanitários. 


\section{Agradecimentos}

Os autores agradecem a Coordenação de Aperfeiçoamento de Pessoal de Nível Superior (CAPES).

\section{Referências}

ABRELPE. Associação Brasileira de Limpeza Pública e Companhias de Resíduos Especiais. Panorama dos Resíduos Sólidos, 2015. Disponível em < http://www.abrelpe.org.br/Panorama/panorama2015.pdf>. Acesso: 15/03/2017.

AEBIOM, 2009. A Biogas Road Map for Europe. Associação de biomassa da Europa.

AGAMUTHU, P. Landfilling in developing countries. Waste Manage. Res. 31, 1-2 Editorial, 2013.

ANEEL. Agência Nacional de Energia Elétrica. Nota informativa: Edital do leilão de Geração A-6 é aprovado pela ANEEL. 2018. Disponível em < http://www.aneel.gov.br/sala-deimprensa-exibicao-2/-/asset_publisher/zXQREz8EVlZ6/content/edital-do-leilao-de-geracao-a-6e-aprovado-pelaaneel/656877/pop_up?_101_INSTANCE_zXQREz8EVlZ6_viewMode=print\&_101_INSTAN CE_zXQREz8EV1Z6_languageId=pt_BR>. Acesso: 20/02/2018.

BARROS, R.M. Tratado sobre resíduos sólidos: gestão, uso e sustentabilidade. Rio de Janeiro: Interciência; Minas Gerais: Acta, 2012. 374p.

BANCO MUNDIAL. Handbook for the Preparation of Landfill Gas to Energy Projects in Latin America and the Caribbean. Conestoga-Rovers \& Associates, 2004. Disponível em < http://www-

wds.worldbank.org/external/default/WDSContentServer/WDSP/IB/2005/08/09/000160016_200 50809131543/Rendered/PDF/332640handbook.pdf>. Acesso: 06/02/2015.

BORGNAKKE, C. e SONTTAG E. R. Fundamentos da Termodinâmica, Série Van Wylen, $8^{\text {a }}$ ed. São Paulo, editora Edgard Blücher Ltda., 2013.

BRASIL, 2012. Plano Nacional de resíduos sólidos. Ministério de meio ambiente. Disponível em

http://www.sinir.gov.br/documents/10180/12308/PNRS_Revisao_Decreto_280812.pdf/e183f0e 7-5255-4544-b9fd-15fc779a3657>. Acesso: 26/09/2017.

BRASIL. Câmara dos Deputados. Nota informativa: Impasse adia para quarta análise de prorrogação de prazo dos lixões, 2017. Disponível em < http://www2.camara.leg.br/camaranoticias/noticias/politica/523907-impasse-adia-para-quartaanalise-de-prorrogacao-de-prazo-dos-lixoes.html >. Acesso: 21/02/2018.

BRASIL. Simulador do Tratamento Tributário e Administrativo das Importações. 2018. Disponível em <http://www4.receita.fazenda.gov.br/simulador/BuscaNCM.jsp>. Acesso: 21/02/2018.

BRANKER, K. PATHAK, M. J. M. PEARCE, J. M. A review of solar photovoltaic levelized cost of electricity. Renewable and Sustainable Energy Reviews. V. 15, p. 4470 - 4482, 2011.

COOLPROP. Table of Contents. 2016. Disponível em: <http://www.coolprop.org/fluid_properties/fluids/Methane.html> Acesso em: 22/02/2018. 
EPE. Empresa de Pesquisa Energética 2015. Livro anual de estatística de energia elétrica. Disponível em: <http://www.epe.gov.br/AnuarioEstatisticodeEnergiaEletrica/Anu\%C3\%A1rio\%20Estat\%C3\% ADstico\%20de\%20Energia\%20E1\%C3\%A9trica\%202015.pdf>. Acesso: 06/06/2017.

IBGE. Instituto Brasileiro de Geografia e Estatistica. 2017. Disponível < https://www.ibge.gov.br/>. Acesso as 06/02/2018.

INSTITUTO NACIONAL DE METEOROLOGIA. Precipitação Acumulada Mensal e Anual (mm). 2015. Consultado em 06 de Março de 2017.

INVESTING. Cotação Dólar Americano - Real Brasileiro do dia 21/02/2018. Disponível em <https://br.investing.com/currencies/usd-brl>. Acesso: 21/02/2018.

JOHARI, A. AHMED, S. I. HASHIM, H. ALKALI, H. RAMLI, M. Economic and environmental benefits of landfill gas from municipal solid waste in Malaysia. Renewable and Sustainable Energy Reviews, V.16, p. 2907-2912, 2012.

LEME, M.M.V. ROCHA, M. H. LORA, E. E. S. VENTURINI, O. J. LOPES, B. M. FERREIRA, C. H. Techno-economic analysis and environmental impact assessment of energy recovery from Municipal Solid Waste (MSW) in Brazil. Resources, Conservation and Recycling. V. 87, p. 8 - 20, 2014.

MORAN, M. J.; SHAPIRO H. N.; BOETTENER D. D.; BAILEY M. B. Fundamentals of Engineering Thermodinamics, $7^{\mathrm{a}}$ ed. Hoboken-New Jersey, publish company Wiley, 2011.

PECORA, V. Implantação de uma Unidade Demonstrativa de Geração de Energia Elétrica a partir do Biogás de Tratamento do Esgoto Residencial da USP - Estudo de Caso. 152 p. Dissertação de Mestrado - Programa Interunidades de Pós-Graduação em Energia da Universidade de São Paulo - PIPGE, 2006.

PEREIRA, I. O. (2018). Tendências do Gás Natural: Análise de Eficiência em Ciclos Combinados - Estudo de Caso. Itajubá, 138 p. Dissertação (Mestrado em Conversão de Energia) - Instituto de Engenharia Mecânica, Universidade Federal de Itajubá.

PMSJC - SECRETARIA MUNICIPAL DA FAZENDA. Dados da Cidade de São José dos Campos, 2017. Disponível em: < http://www.sjc.sp.gov.br/sao_jose/dados_da_cidade.aspx>. Acesso em 13/06/2017.

PNRS. POLÍTICA NACIONAL DE RESÍDUOS SÓLIDOS. Biblioteca Digital da câmara dos Deputados. 2010.

QASIM, S. R. Wastewater Treatment Plants - Planninng design and operation. 2.. Lancaster, Pennsylvania, USA Technomic Publishing Company, 1107 p.,1999.

RANGEL, M. S. BORGES, P. SANTOS, I. F. S. Análise comparativa de custos e tarifas de energias renováveis no Brasil. Revista Brasileira de Energias Renováveis. V.5, n.3, 2016.

SANTOS, I.F.S. VIEIRA, N. D. B. NÓBREGA, L. G. B. BARROS, R. M. TIAGO FILHO, G.L. Assessment of potential biogas production from multiple organic wastes in Brazil: Impact on energy generation, use, and emissions abatement. Resources, Conservation and Recycling. V. 131, P. 54-63, 2018a.

SANTOS, I. F. S. BARROS, R. M. TIAGO FILHO, G. L. Method for determining the number of generators and installed power in LFG energy projects. Submetido a revista Sustainable Cities and Society em 01/2018b. Em revisão. 
SNIS. Sistema Nacional de Informações Sobre Saneamento. 2016. Disponível em < http://app.cidades.gov.br/snisweb/src/Sistema/index>. Acesso: 06/02/2018.

SIEMENS. Turbinas a gás industriais - a abrangente linha de produtos, de 5 a 50 megawatts. 2012. Disponível em <https://www.energy.siemens.com/br/pool/hq/power-generation/gasturbines/downloads/Industrial\%20Gas\%20Turbines/Industrial_Gas_Turbines_POR.pdf > Acesso em: 22/02/2018.

USEPA - United States Environmental Protection Agency (Agência Americana de Proteção Ambiental). Modelo de estimativas de emissões gasosas em aterros sanitários. (LandGEM). Version 3.02 User's Guide. USEPA, 2005. EPA-600/R-05/047.

USEPA. United States Environmental Agency. Landfill Methane Outreach Program (LMOP). Landfill Gas Energy Cost Model: LFGcost-web - User Manual. Washington, USA, 2016. Disponível em <https://www.epa.gov/sites/production/files/2016-12/documents/lfgcostwebv3.1manual_113016.pdf>. Acesso: 03/15/2017. 